\title{
Knee osteoarthritis risk is increased 4-6 fold after knee injury - a systematic review and meta-analysis
}

\author{
Erik Poulsen (D) ,' Glaucia H Goncalves, ${ }^{2}$ Alessio Bricca (D) , ${ }^{3}$ Ewa M Roos (D) , \\ Jonas B Thorlund (D) , ${ }^{1}$ Carsten B Juhl (i) 1,4
}

- Additional material is published online only. To view please visit the journal online (http://dx.doi.org/10.1136/ bjsports-2018-100022).

${ }^{1}$ Department of Sports Science and Clinical Biomechanics, University of Southern Denmark, Odense, Denmark

${ }^{2}$ Department of Physical Therapy, Universidade Federal de Sao Carlos, Sao Carlos, Brazil ${ }^{3}$ Institute of Applied Health Sciences, University of Aberdeen, Aberdeen, United Kingdom ${ }^{4}$ Department of Occupational and Physical Therapy, Gentofte and Herlev Hospitals, Copenhagen University Hospital, Copenhagen, Denmark

\section{Correspondence to} Dr Erik Poulsen, Department of Sports Science and Clinical Biomechanics, University of Southern Denmark, Odense 5230, Denmark; epoulsen@health.sdu.dk

Accepted 29 March 2019 Published Online First 9 May 2019

\section{Check for updates}

(C) Author(s) (or their employer(s)) 2019. No commercial re-use. See rights and permissions. Published by BMJ.

To cite: Poulsen $\mathrm{E}$, Goncalves GH, Bricca A, et al. Br J Sports Med 2019:53:1454-1463.

\section{ABSTRACT}

Objective To estimate knee osteoarthritis (OA) risk following anterior cruciate ligament $(\mathrm{ACL})$, meniscus or combined $A C L$ and meniscus injury.

Design Systematic review and meta-analysis.

Data sources MEDLINE, Embase, SPORTDiscus,

CINAHL and Web of Science until November 2018.

Eligibility criteria for selecting studies Prospective or retrospective studies with at least 2-year follow-up including adults with $A C L$ injury, meniscal injury or combined injuries. Knee OA was defined by radiographs or clinical diagnosis and compared with the contralateral knee or non-injured controls.

Study appraisal and synthesis Risk of bias was assessed using the SIGN50 checklist. ORs for developing knee OA were estimated using random effects metaanalysis.

Results 53 studies totalling $\sim$ million participants were included: 185219 participants with ACL injury, mean age 28 years, 35\% females, $98 \%$ surgically reconstructed; 83267 participants with meniscal injury, mean age 38 years, 36\% females, $22 \%$ confirmed meniscectomy and 73\% unknown; 725362 participants with combined injury, mean age 31 years, 26\% females, $80 \%$ treated surgically. The OR of developing knee OA were 4.2 $\left(95 \% \mathrm{Cl} 2.2\right.$ to $\left.8.0 ; \mathrm{I}^{2}=92 \%\right), 6.3(95 \% \mathrm{Cl} 3.8$ to 10.5 ; $\left.\mathrm{I}^{2}=95 \%\right)$ and $6.4\left(95 \% \mathrm{Cl} 4.9\right.$ to $\left.8.3 ; \mathrm{I}^{2}=62 \%\right)$ for patients with $A C L$ injury, meniscal injury and combined injuries, respectively.

Conclusion The odds of developing knee OA following $A C L$ injury are approximately four times higher compared with a non-injured knee. A meniscal injury and a combined injury affecting both the $\mathrm{ACL}$ and meniscus are associated with six times higher odds compared with a non-injured knee. Large inconsistency (eg, study design, follow-up period and comparator) and few high-quality studies suggest that future studies may change these estimates.

Clinical relevance Patients sustaining a major knee injury have a substantially increased risk of developing knee OA, highlighting the importance of knee injury prevention programmes and secondary prevention strategies to prevent or delay knee OA development. PROSPERO registration number CRD42015016900

\section{INTRODUCTION}

Knee injury is one of the strongest risk factors for development of knee osteoarthritis (OA). ${ }^{12}$ However, the relative importance of different types of knee injuries such as ACL injury and meniscal injury for knee OA risk is less clear. Previous systematic reviews report higher risk of knee OA in patients with combined ACL and meniscal injury compared with patients with isolated ACL injury. ${ }^{34}$ Common for previous systematic reviews were that these did not quantitatively assess the risk of OA but relied on a narrative or 'best evidence synthesis' approach and did not include studies on patients with isolated meniscal injury, precluding any definitive conclusions about the relative importance of ACL injury and meniscal injury on subsequent risk of knee OA development. ${ }^{3}$

We performed an updated comprehensive systematic review and meta-analysis to ascertain the risk of knee OA development in adults following isolated ACL injury, isolated meniscal injury or combined ACL and meniscus injury.

\section{MATERIALS AND METHODS}

This systematic review was performed according to the guidelines from the Cochrane collaboration and followed the Preferred Reporting Items for Systematic Reviews and Meta-analysis (PRISMA) guidelines. 56 The study protocol is available online: http://www.crd.york.ac.uk/PROSPERO, CRD42015016900).

\section{Literature search}

The senior author (CBJ) developed a comprehensive search strategy, and a systematic search for published literature was performed in the electronic databases MEDLINE via PubMed, Embase via Ovid, SPORTDiscus, CINAHL via EBSCO and Web of Science up until 1 May 2015 by GHG. Two authors (GHG and EP) screened the titles, abstracts and full texts. An updated search was performed up to 1 November 2018. Furthermore, a supplementary manual search was performed based on the reference lists from the included studies and systematic reviews/meta-analyses by the first author (EP).

Where possible, all terms were searched as keywords (Medical Subject Headings $(\mathrm{MeSH})$ ) and text words in titles and abstracts to create three separate filters, which were then combined. These filters were: (1) knee injury: "menisc", "anterior cruciate ligament" or "knee injury", (2) knee OA: "radiographic", "radiological", "osteoarthritis", "degenerative arthritis" or "osteoarthrosis" and (3) risk factors: "causality", "risk factors" or "prognosis". The complete search strategy for all included databases is presented in detail in online supplementary appendix 1 . No restriction on year of publication was applied, and only studies published in English, Norwegian, Swedish or Danish were included. 


\section{Eligibility criteria}

We included studies meeting the following criteria: (1) design: prospective or retrospective designs with a follow-up time of a minimum of 2 years after knee injury/surgery. A minimum of 2 years was chosen as radiographic OA development has been demonstrated at 2 years following ACL reconstruction combined with meniscal surgery ${ }^{7}$; (2) population: participants having an ACL injury, meniscal injury, a combination of ACL injury and meniscal injury and a mean minimum age of 18 years at the time of injury. Studies including patients reported to have OA at time of injury were excluded; (3) comparison: risk of the injured knee needed to have been compared with either the contralateral leg or a non-injured control group; (4) outcome: development of knee OA defined as: (1) radiographic knee OA of the tibiofemoral joint according to either Kellgren \& Lawrence classification $\geq 2$, Ahlbäck grading scale $\geq 1$, International Knee Documentation Committee (IKDC) radiographic scale C and D, Fairbank $\geq 2$ or other radiographic scoring systems as well as reporting of total knee arthroplasty (TKA) due to OA, (2) symptomatic knee OA (ie, defined by criteria such as those developed by the American College of Rheumatology) or (3) self-reported knee OA. ${ }^{8-10}$

\section{Selection of studies}

Following the removal of duplicates from the initial search, two authors (GHG/EP) independently screened the articles by title and abstract to identify relevant studies. Full text of all abstracts considered relevant by either of these reviewers was obtained and screened independently for eligibility by both. The full-text studies were assessed independently by the same two authors and reasons for exclusion were listed. For the updated search, the authors EP and AB repeated these procedures.

\section{Data extraction}

The following data were extracted from the included studies: authors, publication year, country of origin, study design, number of participants at follow-up (with or without OA), participant characteristics (ie, age at time of injury/surgery, sex, body mass index (BMI) and activity level prior to injury), criteria for definition of symptomatic and/or radiographic OA, comparison group (ie, healthy control or contralateral leg), type of knee injury (ie, ACL injury, meniscal injury or combination of ACL and meniscal injury) and other knee injuries in addition (such as medial or lateral collateral ligament injury, posterior cruciate ligament and chondral injury and so on). When data could be extracted for more than one injury type, the OR for OA development was estimated for each injury type by comparing each injury group with the control group (eg, ACL injury vs control and combined vs control). ${ }^{6}$ Hence, the reported ORs reflect the odds of developing knee OA after an ACL, meniscal or combined injury in comparison with a non-injured knee. Furthermore, data were extracted involving intervention (surgical, non-surgical or no intervention) and time to follow-up.

\section{Risk of bias assessment}

Two of the authors (EP/CBJ) independently evaluated risk of bias of included studies using the SIGN50 checklists. ${ }^{11}$ Authors EP and $\mathrm{AB}$ performed the same task for the updated search. The checklist evaluates internal validity with the domains: focused research question, selection of subjects, assessment, confounding and statistical analysis and an overall study assessment. The overall assessment of included studies was classified as high quality, acceptable quality or unacceptable quality. ${ }^{11}$ Prior to risk of bias assessment, the evaluating authors familiarised themselves with the guidelines for scoring each of the criteria as detailed in SIGN50. Disagreement was solved by discussion. The SIGN50 checklist is included in online supplementary appendix 2 .

\section{Strategy for data synthesis}

As studies with 'clean' populations of isolated ACL tears are rare, we categorised study participants into three groups based on injury type: (1) the ACL group included studies with participants having isolated ACL injury and allowing for up to $20 \%$ of participants having a concurrent meniscal injury. (2) The meniscus group included studies with participants having an isolated meniscal injury and allowing for up to $20 \%$ of participants having a concurrent ACL injury. (3) The combined group included studies where more than $20 \%$ of participants were reported to have the combination of ACL and meniscus injury. Meta-analysis was applied based on the logarithmic transformed OR of developing knee OA in subjects with isolated ACL injury, meniscal injury and a combination of ACL and meniscal injury, respectively. Data from adjusted analyses of included studies were extracted if available. Meta-analysis using random effect models was applied as large clinical heterogeneity was expected in participant characteristics, comparator group, follow-up time and the definitions of knee OA. Between-study variance was estimated as Tau-squared, ${ }^{12}$ and heterogeneity was calculated as the I-squared ( $\mathrm{I}^{2}$ statistics $),{ }^{13}$ measuring the proportion of inconsistency in the combined estimates due to between-study heterogeneity. ${ }^{14}$ An $\mathrm{I}^{2}$ equal to $0 \%$ indicates minimal inconsistency, and an $\mathrm{I}^{2}$ equal to $100 \%$ indicates maximal inconsistency between individual study results. Covariates are defined as variables able to reduce the Tau-squared when included in the analysis. Additional meta-regression analyses were performed to investigate the impact of physical activity level prior to injury and time to follow-up. Physical activity level was evaluated by dichotomising reported Tegner scores prior to injury (Tegner $<7=$ low to moderate activity prior to injury and Tegner $\geq 7=$ high activity). Time to follow-up was used as a continuous variable (year) and also dichotomised into $\leq 10$ years and $>10$ years. Risk of small study bias was assessed by visual inspection of funnel plots of included studies in the three groups.

Some studies, examining OA risk following ACL or meniscal injuries did not specifically report patient OA status at time of injury. Therefore, we performed a sensitivity analysis excluding studies with no information about baseline OA status and a mean patient age of 35 years or older as some of these patients could have OA at time of injury. In the analysis, we retained studies specifically reporting exclusion of patients with $\mathrm{OA}$ at time of injury and studies not reporting OA status at time of injury but a mean patient age under 35 years, as these patients are unlikely to have $\mathrm{OA}$ at time of injury. Furthermore, we performed a sensitivity analysis changing the cut-offs for isolated ACL and meniscal injury allowing only $10 \%$ concurrent meniscal or ACL injury and an analysis calculating the overall risk (OR) stratified by uninjured knee versus a healthy control group as comparator.

\section{RESULTS}

Following the initial literature search and after duplicates were removed, 8416 studies were reviewed by title and abstract, 341 studies were included for full-text screening and 53 papers were included in the meta-analysis. The updated search including 1 November 2018 revealed seven new studies for inclusion in the meta-analysis. The PRISMA flow chart including reason for fulltext exclusion is attached in figure 1. 


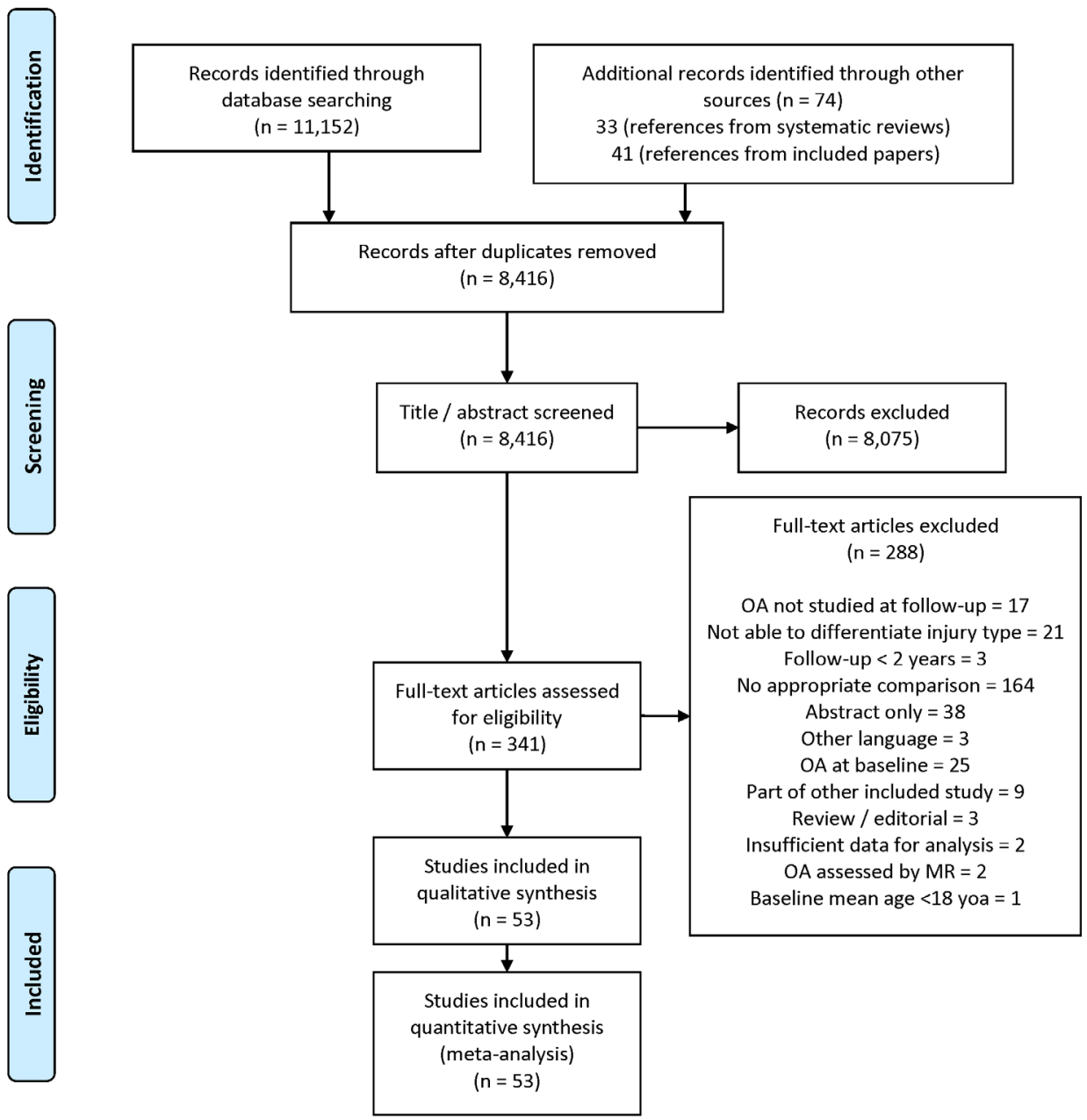

From: Moher D, Liberati A, Tetzlaff J, Altman DG, The PRISMA Group (2009). Preferred Reporting /tems for Systematic Reviews and MetaAnalyses: The PRISMA Statement. PLoS Med 6(7): e1000097. doi:10.1371/journal.pmed1000097

For more information, visit www.prisma-statement.org.

Figure 1 PRISMA flow chart of study selection. OA, osteoarthritis; PRISMA, Preferred Reporting Items for Systematic Reviews and Meta-analysis.

\section{Study characteristics}

Of the 53 included studies, 11 investigated isolated ACL injury (185 219 participants, 35\% females, mean age 28.1 years at time of injury), 22 meniscal injury (83 267 participants, 36\% females, mean age 38.1 years at time of injury) and 25 combined injury (725 362 participants, 26\% females, mean age 30.5 years at time of injury). One study reported both ACL and meniscal injuries separately, two papers reported isolated ACL and combined injuries separately and two studies reported isolated meniscal and combined injuries separately. Additional details of included studies are listed in table 1.

Forty-two studies used the opposite knee as a control, and 11 studies involved an age-matched, and sex-matched control group or a reference population of similar mean age and sex distribution. The knee OA outcome was based on knee radiographs for $91 \%$ of the studies $(n=48)$, one study used selfreported OA, two studies used physician-diagnosed OA and two studies reported TKA due to knee OA. Radiographic OA defined by a cut-off of Kellgren $\&$ Lawrence grade $\geq 2$ was reported in 19 studies, by Albäck score $\geq 1$ in 10 studies, by IKDC score (C and D) in 9 studies and by Fairbank score $\geq 2$ in 6 studies. Four studies used other radiographic grading systems.

The risk of OA development was assessed regardless of treatment strategy (surgery/non-surgery/not reported) in all three groups. For the isolated ACL injury group, 98\% of participants had arthroscopic ACL reconstruction and for the remaining 2\%, the treatment was not specified. For the meniscal injury group, $22 \%$ had meniscal surgery, the treatment strategy was not reported for $73 \%$ of participants, and treatment was non-surgical for 5\% of participants. In the combined injury group, $26 \%$ of participants had received ACL reconstruction as the primary surgery, meniscectomy was reported as the primary surgery for $54 \%$ of participants and for $20 \%$ of participants treatment was not specified.

\section{Risk of knee OA}

The OR for developing knee OA in comparison with a non-injured knee following ACL injury and meniscal injury were 4.2 (95\% CI 2.2 to $\left.8.0 ; \mathrm{I}^{2}=92 \%\right)$ and $6.3\left(95 \%\right.$ CI 3.8 to $\left.10.5 ; \mathrm{I}^{2}=95 \%\right)$, respectively. The odds of developing OA following a combined 


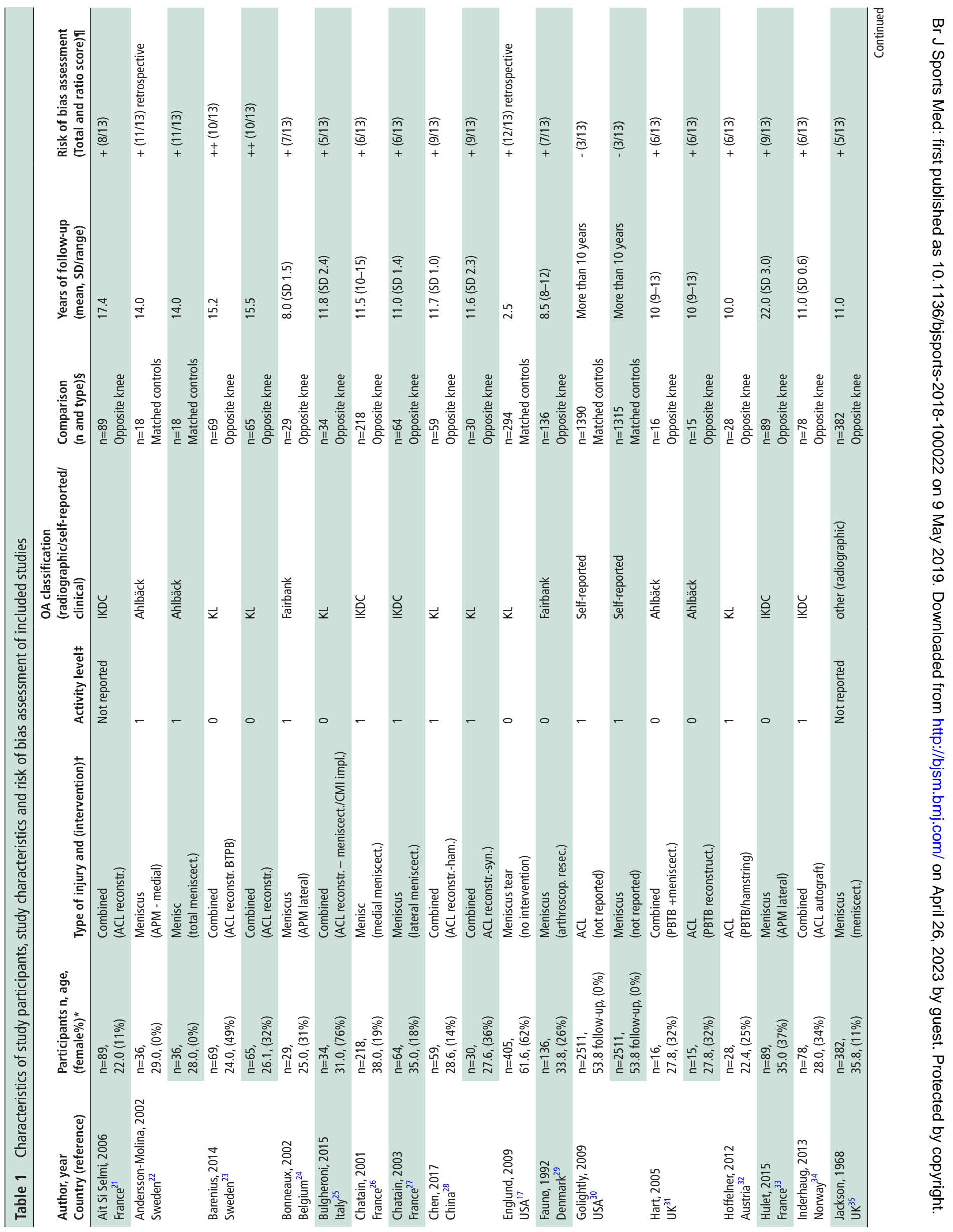




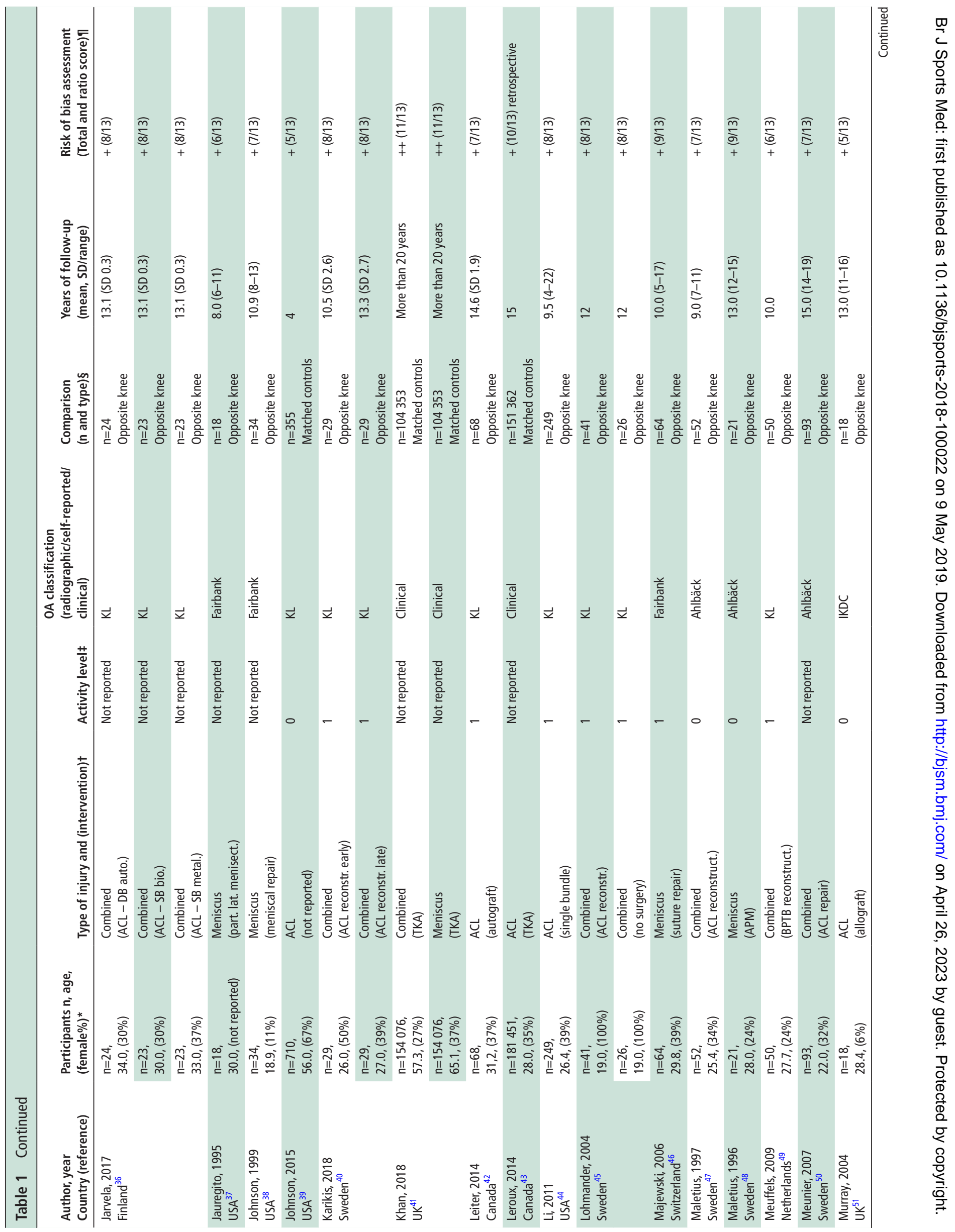




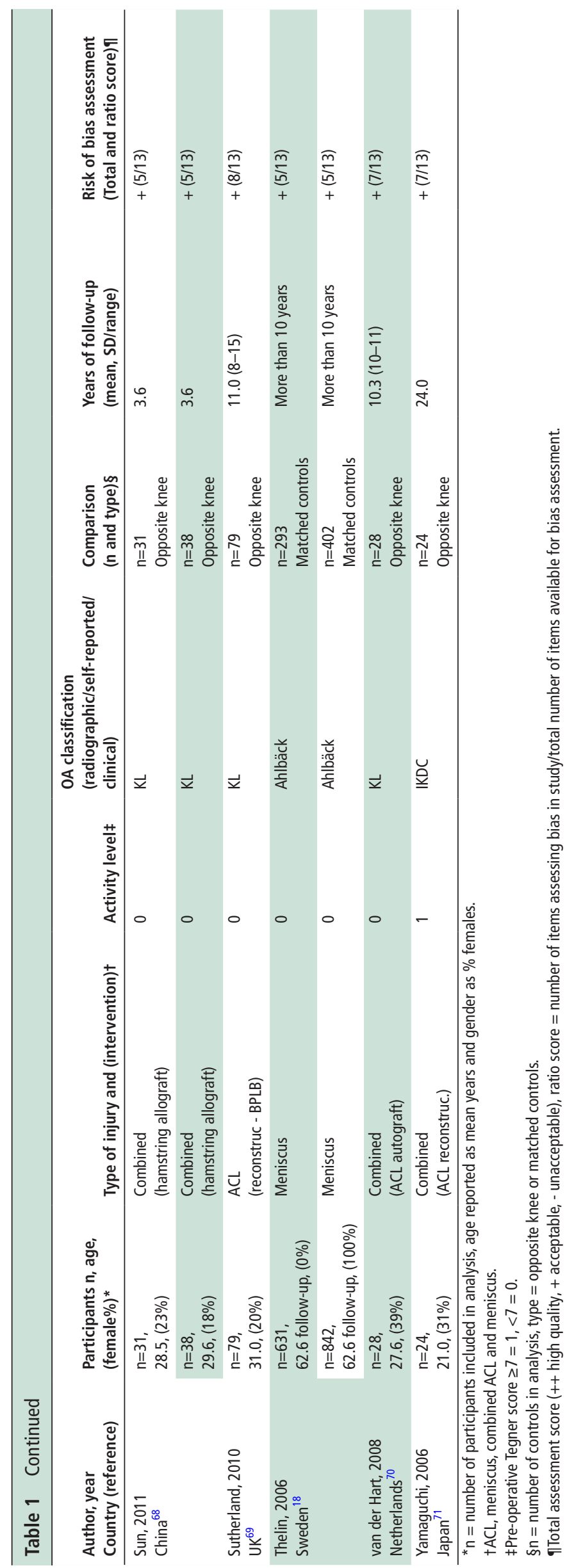

ACL and meniscal injury was 6.4 (95\% CI 4.9 to $\left.8.3 ; \mathrm{I}^{2}=62 \%\right)$ times higher compared with a non-injured knee (figure 2 ).

Sensitivity analyses lowering the threshold for overlap between study types from $20 \%$ to $10 \%$, excluding studies with patient mean age of 35 years or older and not reporting OA status at time of injury as well as using the opposite knee versus using an uninjured control did not markedly change the estimates (online supplementary appendix 3).

\section{Physical activity}

Overall, 40 studies (75\%) reported activity levels primarily using the Tegner score. Activity level (low-moderate vs high) prior to injury did not influence the risk of knee OA development, OR 0.85 (95\% CI 0.51 to 1.41 ).

\section{Length of follow-up}

For $80 \%$ of the studies, the follow-up period was more than 10 years. The analysis indicated that studies with a longer time to follow-up had a significantly higher probability of reporting OA development (slope 1.049 , 95\% CI 1.009 to 1.090) (figure 3). Furthermore, categorising into follow-up periods into less than or more than 10 years, longer follow-up time (ie, 10 years or more) was associated with higher risk of developing knee OA (OR 1.46 (95\% CI 0.95 to 2.26)).

\section{Risk of bias}

Four studies were rated as having low risk of bias, 48 (91\%) studies as having moderate risk of bias and 1 study as having high risk of bias. Ten studies were prospective and 43 were retrospective. Risk of bias assessments of individual studies are given in table 1.

Visual inspection of funnel plots of included studies (figure $4 \mathrm{~A}-\mathrm{C}$ ) indicated no risk of small study bias for the ACL or meniscal groups, whereas a risk of small study bias was present in the combined injury group.

No subgroup analyses were performed for BMI, baseline pain, surgery versus no surgery and additional injury due to insufficient data. BMI was reported in only 11 studies (21\%), of which six reported BMI at follow-up. Additional injuries (collateral ligament and/or chondral) were reported for nine studies (17\%). Pain level at baseline were reported for only one study.

\section{DISCUSSION}

In comparison with previous studies, this study provides pooled estimates of the additional risk of knee OA following different types of knee injuries in comparison with a non-injured knee. The results of the current synthesis confirm that both ACL and meniscal injuries are strong individual risk factors for the development of knee OA at 10 or more years following injury. At the same time, the results indicate that a meniscal lesion, isolated or in combination with an ACL injury is an even more important risk factor than an isolated surgically reconstructed ACL injury. Our results suggest that OA risk after knee injury is time dependent and increases with longer follow-up time.

This is the first systematic review to provide pooled estimates of OA risk following three different types of knee injuries. However, it should be noted that the populations sustaining these different types of injuries differ with regard to age. Individuals sustaining an injury involving the ACL were on average 28 and 31 years of age, while those with an isolated meniscal injury were on average 38 years. The difference in age indicates that individuals sustaining an injury involving the ACL 


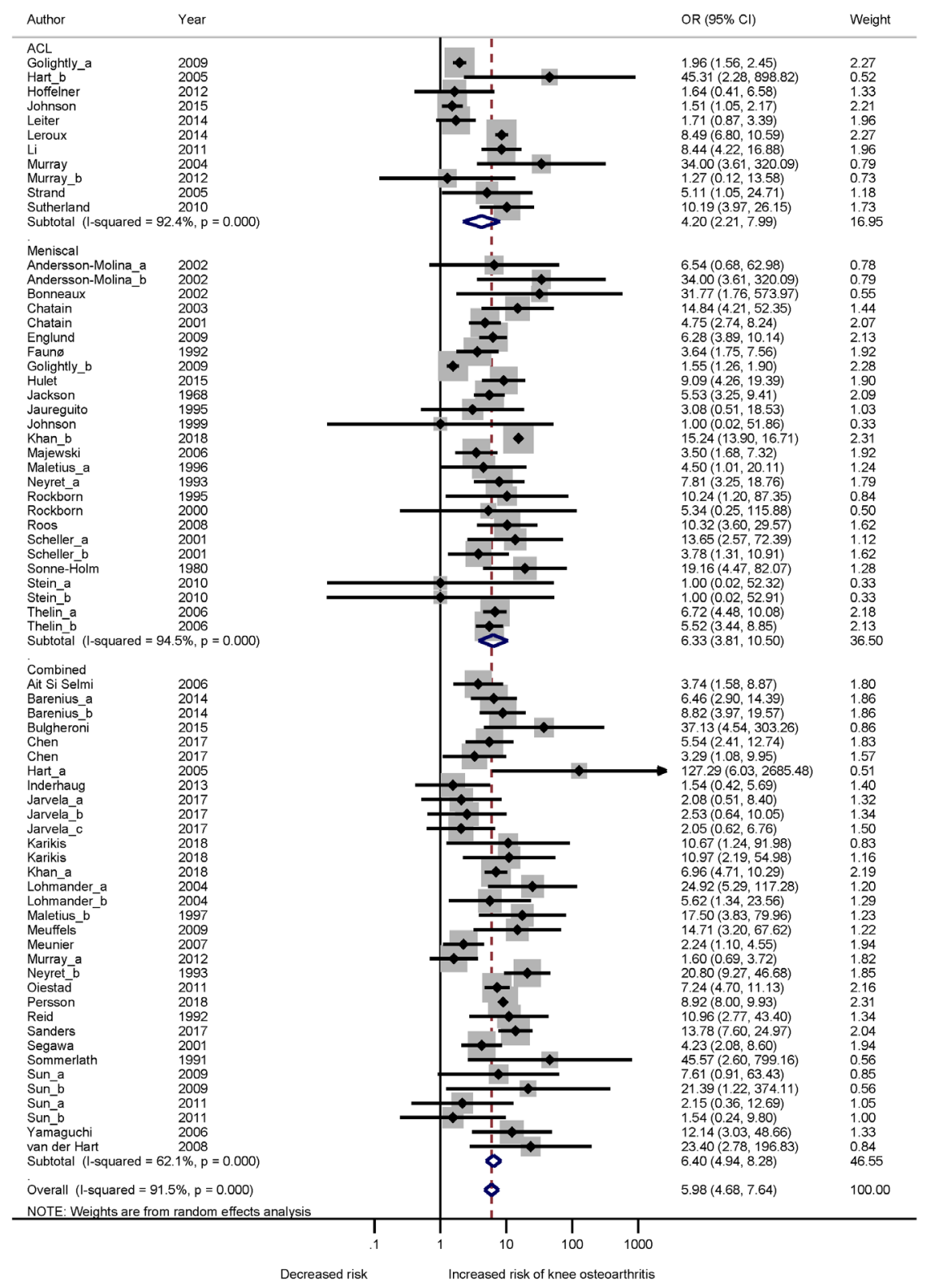

Figure 2 Forest plot showing ORs for OA development following ACL, meniscal and combined knee injury compared with a non-injured control knee.

are representative of young adults sustaining a trauma, typically during sport, whereas the group with isolated meniscal injury is more diverse.

Meniscal injury can be traumatic, for example, commonly a vertical longitudinal tear sustained to a healthy meniscus, or degenerative, for example, commonly horizontal and complex tears having a sudden or insidious onset. Both types of injury are associated with an increased risk of $\mathrm{OA},{ }^{15}$ but the mechanisms may differ. For the traumatic tear, it has been suggested to be the tear itself or the frequent loss of tissue from surgical removal and the (resulting) change in joint loading and biomechanics that drive OA development, while the degenerative tear in the middle-aged and older individual has been suggested to be part of the OA process itself. ${ }^{16}$ In our synthesis, one populationbased study from the USA ${ }^{17}$ and one study where cases were systematically identified from radiology departments in one geographical area in Sweden, ${ }^{18}$ both with mean ages slightly over 60 years, contribute to the higher mean age in the meniscal subgroup. In the US population-based study, meniscal injury was confirmed by MRI at a mean age of 61 years, indicating the meniscal tears were degenerative by nature. In the Swedish study reporting a physician-confirmed meniscal injury, the presence of meniscus injury was self-reported retrospectively, and the age at time of injury and the most likely type of meniscal tear (traumatic or degenerative) are therefore unknown.

\section{Comparison with previous literature}

Several systematic reviews and meta-analyses have reported on the risk of developing knee OA following a knee injury, but 


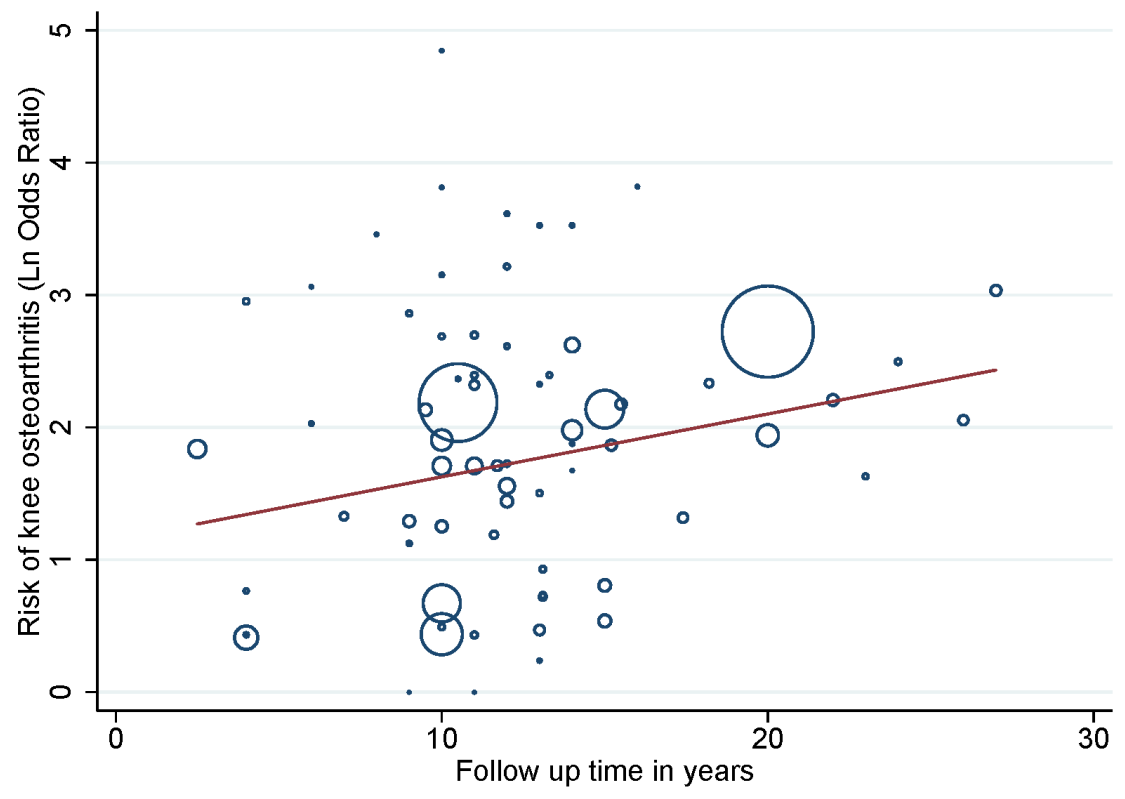

Figure 3 Metaregression analysis of impact of follow-up time on the OR of OA development. Weight of individual studies is indicated by the size of the circles, a study with high precision (typically more participants - larger circle) has greater weight. OA, osteoarthritis.

direct comparison is restricted by difference in eligibility criteria such as primary study aim, pooling of injury types, intervention investigated, comparator, follow-up time, outcomes and type of analyses. However, overall an increased risk of knee OA is reported following major knee injuries. Pooling all knee injury types, Silverwood et al. and Muthuri et al. found three and four times higher odds of developing knee OA after knee injury in comparison ith an uninjured knee but with large heterogeneity in estimates. ${ }^{12}$ Claes et al. ${ }^{19}$ included ACL reconstructed knees with or without meniscectomy and found an almost four times higher odds of OA development when the surgery included both the ACL and the meniscus in comparison with ACL reconstruction alone, but there was no comparison to an uninjured knee. Similar to the present study, the OA risk increased with time to follow-up. We only identified one recent systematic review reporting on long-term prevalence of radiographic OA $(>10$ years) after arthroscopic partial meniscectomy; however, no estimate for comparison was available as no meta-analysis was performed. ${ }^{20}$

\section{Limitations}

An important limitation is that some of the included studies did not specifically report patient knee OA status at time of injury/ surgery. We decided to include these studies in this systematic review and only exclude studies specifically reporting presence of OA at time of injury/surgery. Thus, some studies may have included patients with pre-existing $\mathrm{OA}$ at time of inclusion. To take this into account, we performed a sensitivity analysis including only studies specifically reporting no OA at time of injury/surgery and studies with patient mean age lower than 35 years (these patients would not be expected to have preexisting OA). The estimates from this sensitivity analysis only slightly reduced the risk in the meniscal and combined group.

Few studies were restricted to isolated ACL or meniscal injury. We allowed up to $20 \%$ overlap between injury types but conducted a sensitivity analysis allowing only up to $10 \%$ overlap between injury types, which yielded similar estimates of OA risk. Another limitation is that comparison between surgery and non-surgical treatment strategies was not possible, since treatment strategy was unknown for $73 \%$ and $20 \%$, respectively, of the meniscal and combined groups. For the ACL group, 98\% had had ACL reconstruction surgery, and the results are generalisable to those having had surgical reconstruction but not those treated without surgery. The variation in participant characteristics, study size, type of study (retrospective vs prospective and case-control vs randomised
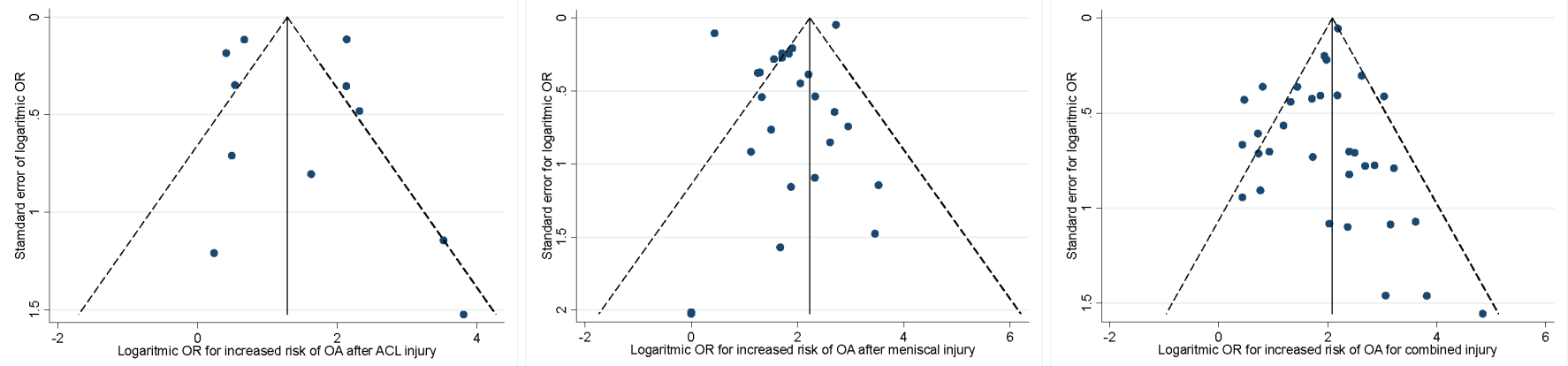

Figure 4 Funnel plots for OA development of included studies investigating risk of small study bias stratified by injury type. OA, osteoarthritis. 
controlled trial), type of intervention and outcomes contribute to the high inconsistencies seen in the meta-analyses. However, different sensitivity analyses showed consistent results, indicating a high risk of knee OA development following the three types of injury.

\section{Implications for research}

Within the last year, several large high-quality registry studies have been published investigating the risk of OA development following meniscal and ACL injuries but using different definitions of OA. To further improve the quality in this area, future research should focus on large prospective cohorts with high follow-up rates reporting both patient-reported outcomes, imaging and the combination thereof to allow for comparison across studies using different OA definitions. Increased implementation of primary and secondary prevention programmes is urgently needed to reduce the number of knee injuries and the future increasing burden of OA.

\section{CONCLUSIONS}

This meta-analysis provides estimates for the risk of knee OA development following three knee injury types compared with a non-injured knee. An isolated ACL injured knee is associated with a four times higher odds of subsequent knee OA development compared with a non-injured knee. When sustaining an isolated meniscal injury or an injury affecting both the ACL and meniscus, the odds increase to sixfold compared with a noninjured knee, indicating the injured meniscus plays an important role in OA development. However, due to great between-study variation in design and outcomes collected and the lack of prospective studies with high-follow up rates, future high-quality studies may change the estimates.

\section{What is already known}

- Major knee injury such as $A C L$ or meniscus injury are important risk factors for developing osteoarthritis (OA), but their relative importance is unknown.

\section{What are the new findings}

- Compared with an uninjured knee, patients with a meniscal injury, isolated or combined with an ACL injury have a higher relative risk for development of knee $\mathrm{OA}$ than patients with an isolated $\mathrm{ACL}$ injury.

Contributors JBT, EMR and CBJ conceived and planned the study. GHG and CBJ performed the searches, and $E P, G H G$ and $A B$ performed the screening of papers. $E P$, $G H G, A B$ and $C B J$ did the data extraction. $C B J$ independently performed the analyses, and EP, GHG, EMR, JBT and CBJ contributed to the draft of the manuscript. All authors participated in reviewing and editing the manuscript, and all authors have read and approved the final version.

Funding The authors have not declared a specific grant for this research from any funding agency in the public, commercial or not-for-profit sectors.

Competing interests None declared.

Patient consent for publication Not required.

Provenance and peer review Not commissioned; externally peer reviewed.

ORCID iDs

Erik Poulsen http://orcid.org/0000-0002-3837-5452

Alessio Bricca http://orcid.org/0000-0001-9717-918X

Ewa M Roos http://orcid.org/0000-0001-5425-2199

Jonas B Thorlund http://orcid.org/0000-0001-7789-8224

Carsten B Juhl 0000-0001-8456-5364

\section{REFERENCES}

1. Silverwood V, Blagojevic-Bucknall M, Jinks C, et al. Current evidence on risk factors for knee osteoarthritis in older adults: a systematic review and meta-analysis. Osteoarthritis Cartilage 2015;23:507-15.

2. Muthuri SG, McWilliams DF, Doherty M, et al. History of knee injuries and knee osteoarthritis: a meta-analysis of observational studies. Osteoarthritis Cartilage 2011;19:1286-93.

3. Øiestad BE, Engebretsen L, Storheim K, et al. Knee osteoarthritis after anterior cruciate ligament injury: a systematic review. Am I Sports Med 2009;37:1434-43.

4. van Meer BL, Meuffels DE, van Eijsden WA, et al. Which determinants predict tibiofemoral and patellofemoral osteoarthritis after anterior cruciate ligament injury? A systematic review. Br J Sports Med 2015;49:975-83.

5. Moher $\mathrm{D}$, Liberati A, Tetzlaff J, et al. Preferred reporting items for systematic reviews and meta-analyses: the PRISMA statement. Ann Intern Med 2009;151:264-9.

6. Higgins JPTG S, ed. Cochrane Handbook for systematic reviews of interventions. The Cochrane collaboration, 2011.

7. Jones $\mathrm{MH}$, Spindler KP, Fleming BC, et al. Meniscus treatment and age associated with narrower radiographic joint space width 2-3 years after $\mathrm{ACL}$ reconstruction: data from the moon onsite cohort. Osteoarthritis Cartilage 2015;23:581-8.

8. Altman R, Asch E, Bloch D, et al. Development of criteria for the classification and reporting of osteoarthritis. Classification of osteoarthritis of the knee. Diagnostic and therapeutic criteria Committee of the American rheumatism association. Arthritis Rheum 1986;29:1039-49.

9. Altman RD. Criteria for the classification of osteoarthritis of the knee and hip. Scand J Rheumatol Supp/ 1987;65:31-9.

10. March LM, Schwarz JM, Carfrae BH, et al. Clinical validation of self-reported osteoarthritis. Osteoarthritis Cartilage 1998;6:87-93.

11. Scottish Intercollegiate Guidelines Network HIS. Methodology checklist for systematic reviews and meta-analyses Edinburgh. Scotland: Scottish Intercollegiate Guidelines Network, 2012.

12. Thompson SG, Higgins JPT. Treating individuals 4: can meta-analysis help target interventions at individuals most likely to benefit? Lancet 2005;365:341-6.

13. Higgins JPT, Thompson SG. Quantifying heterogeneity in a meta-analysis. Stat Med 2002;21:1539-58.

14. Higgins JPT, Thompson SG, Deeks JJ, et al. Measuring inconsistency in meta-analyses. BMJ 2003;327:557-60.

15. Englund M, Roos EM, Lohmander LS. Impact of type of meniscal tear on radiographic and symptomatic knee osteoarthritis: a sixteen-year followup of meniscectomy with matched controls. Arthritis Rheum 2003;48:2178-87.

16. Englund M, Roemer FW, Hayashi D, et al. Meniscus pathology, osteoarthritis and the treatment controversy. Nat Rev Rheumatol 2012;8:412-9.

17. Englund M, Guermazi A, Roemer FW, et al. Meniscal tear in knees without surgery and the development of radiographic osteoarthritis among middleaged and elderly persons: The Multicenter osteoarthritis study. Arthritis Rheum 2009;60:831-9.

18. Thelin N, Holmberg S, Thelin A. Knee injuries account for the sports-related increased risk of knee osteoarthritis. Scand J Med Sci Sports 2006;16:329-33.

19. Claes $S$, Hermie L, Verdonk R, et al. Is osteoarthritis an inevitable consequence of anterior cruciate ligament reconstruction? A meta-analysis. Knee Surg Sports Traumatol Arthrosc 2013;21:1967-76.

20. Petty CA, Lubowitz JH. Does arthroscopic partial meniscectomy result in knee osteoarthritis? A systematic review with a minimum of 8 years' follow-up. Arthroscopy 2011;27:419-24

21. Ait Si Selmi T, Fithian D, Neyret P. The evolution of osteoarthritis in 103 patients with ACL reconstruction at 17 years follow-up. The Knee 2006;13:353-8.

22. Andersson-Molina H, Karlsson H, Rockborn P. Arthroscopic partial and total meniscectomy: a long-term follow-up study with matched controls. Arthroscopy 2002;18:183-9.

23. Barenius B, Ponzer $S$, Shalabi A, et al. Increased risk of osteoarthritis after anterior cruciate ligament reconstruction: a 14-year follow-up study of a randomized controlled trial. Am J Sports Med 2014;42:1049-57.

24. Bonneux I, Vandekerckhove B. Arthroscopic partial lateral meniscectomy long-term results in athletes. Acta Orthop Belg 2002;68:356-61.

25. Bulgheroni P, Murena L, Ratti C, et al. Follow-up of collagen meniscus implant patients: clinical, radiological, and magnetic resonance imaging results at 5 years. Knee 2010;17:224-9.

26. Chatain $F$, Robinson $A H$, Adeleine $P$, et al. The natural history of the knee following arthroscopic medial meniscectomy. Knee Surg Sports Traumatol Arthrosc 2001;9:15-18.

27. Chatain $F$, Adeleine $P, C$ Chambat $P$, et al. A comparative study of medial versus lateral arthroscopic partial meniscectomy on stable knees: 10-year minimum follow-up. Arthroscopy 2003;19:842-9.

28. Chen T, Zhang P, Chen J, et al. Long-term outcomes of anterior cruciate ligament reconstruction using either Synthetics with remnant preservation or hamstring autografts: a 10-year longitudinal study. Am J Sports Med 2017;45:2739-50.

29. Faunø P, Nielsen AB. Arthroscopic partial meniscectomy: a long-term follow-up. Arthroscopy 1992;8:345-9. 
30. Golightly YM, Marshall SW, Callahan LF, et al. Early-onset arthritis in retired National Football League players. J Phys Act Health 2009;6:638-43.

31. Hart AJ, Buscombe J, Malone A, et al. Assessment of osteoarthritis after reconstruction of the anterior cruciate ligament: a study using single-photon emission computed tomography at ten years. J Bone Joint Surg Br 2005;87:1483-7.

32. Hoffelner $T$, Resch $H$, Moroder $P$, et al. No increased occurrence of osteoarthritis after anterior cruciate ligament reconstruction after isolated anterior cruciate ligament injury in athletes. Arthroscopy 2012;28:517-25.

33. Hulet C, Menetrey J, Beaufils P, et al. Clinical and radiographic results of arthroscopic partial lateral meniscectomies in stable knees with a minimum follow up of 20 years. Knee Surg Sports Traumatol Arthrosc 2015;23:225-31.

34. Inderhaug E, Strand T, Fischer-Bredenbeck C, et al. Long-term results after reconstruction of the $\mathrm{ACL}$ with hamstrings autograft and transtibial femoral drilling. Knee Surg Sports Traumatol Arthrosc 2013;21:2004-10.

35. Jackson JP. Degenerative changes in the knee after meniscectomy. BMJ 1968;2:525-7.

36. Järvelä S, Kiekara T, Suomalainen $P$, et al. Double-Bundle versus Single-Bundle anterior cruciate ligament reconstruction: a prospective randomized study with 10-year results. Am J Sports Med 2017:45:2578-85.

37. Jaureguito JW, Elliot JS, Lietner T, et al. The effects of arthroscopic partial lateral meniscectomy in an otherwise normal knee: a retrospective review of functional, clinical, and radiographic results. Arthroscopy 1995;11:29-36.

38. Johnson MJ, Lucas GL, Dusek JK, et al. Isolated arthroscopic meniscal repair: a longterm outcome study (more than 10 years). Am J Sports Med 1999;27:44-9.

39. Johnson VL, Kwoh CK, Guermazi A, et al. Loss of anterior cruciate ligament integrity and the development of radiographic knee osteoarthritis: a sub-study of the osteoarthritis initiative. Osteoarthritis and Cartilage 2015;23:882-7.

40. Karikis I, Åhlén M, Sernert N, et al. The long-term outcome after early and late anterior cruciate ligament reconstruction. Arthroscopy 2018;34:1907-17.

41. Khan $T$, Alvand $A$, Prieto-Alhambra $D$, et al. ACL and meniscal injuries increase the risk of primary total knee replacement for osteoarthritis: a matched case-control study using the clinical Practice Research Datalink (CPRD). Br J Sports Med 2018 (Epub ahead of print: 2019/01/15).

42. Leiter JRS, Gourlay R, McRae S, et al. Long-term follow-up of ACL reconstruction with hamstring autograft. Knee Surg Sports Traumatol Arthrosc 2013;17:1061-9.

43. Leroux T, Ogilvie-Harris D, Dwyer T, et al. The risk of knee arthroplasty following cruciate ligament reconstruction: a population-based matched cohort study. J Bone Joint Surg Am 2014;96:2-10.

44. Li RT, Lorenz S, Xu Y, et al. Predictors of radiographic knee osteoarthritis after anterior cruciate ligament reconstruction. Am J Sports Med 2011;39:2595-603.

45. Lohmander LS, Östenberg A, Englund M, et al. High prevalence of knee osteoarthritis, pain, and functional limitations in female soccer players twelve years after anterior cruciate ligament injury. Arthritis \& Rheumatism 2004:50:3145-52.

46. Majewski M, Stoll R, Widmer $H$, et al. Midterm and long-term results after arthroscopic suture repair of isolated, longitudinal, vertical meniscal tears in stable knees. Am J Sports Med 2006;34:1072-6.

47. Maletius W, Gillquist J. Long-term results of anterior cruciate ligament reconstruction with a Dacron prosthesis. the frequency of osteoarthritis after seven to eleven years. Am J Sports Med 1997;25:288-93

48. Maletius W, Messner K. The effect of partial meniscectomy on the long-term prognosis of knees with localized, severe chondral damage. Am J Sports Med 1996;24:258-62.

49. Meuffels DE, Favejee MM, Vissers MM, et al. Ten year follow-up study comparing conservative versus operative treatment of anterior cruciate ligament ruptures. a matched-pair analysis of high level athletes. Br J Sports Med 2009;43:347-51.

50. Meunier A, Odensten M, Good L. Long-term results after primary repair or nonsurgical treatment of anterior cruciate ligament rupture: a randomized study with a 15-year follow-up. Scand J Med Sci Sports 2007;17:230-7.
51. Murray AW, Macnicol MF. 10-16 year results of Leeds-Keio anterior cruciate ligament reconstruction. The Knee 2004:11:9-14.

52. Murray JR, Lindh AM, Hogan NA, et al. Does anterior cruciate ligament reconstruction lead to degenerative disease?: Thirteen-year results after bone-patellar tendon-bone autograft. Am J Sports Med 2012;40:404-13.

53. Neyret P, Donell ST, Dejour H. Results of partial meniscectomy related to the state of the anterior cruciate ligament. Review at 20 to 35 years. J Bone Joint Surg $\mathrm{Br}$ 1993; 75:36-40.

54. Oiestad BE, Holm I, Engebretsen L, et al. The association between radiographic knee osteoarthritis and knee symptoms, function and quality of life 10-15 years after anterior cruciate ligament reconstruction. Br J Sports Med 2011;45:583-8.

55. Persson F, Turkiewicz A, Bergkvist $D$, et al. The risk of symptomatic knee osteoarthritis after arthroscopic meniscus repair vs partial meniscectomy vs the general population. Osteoarthritis Cartilage 2018;26:195-201

56. Reid JS, Hanks GA, Kalenak A, et al. The Ellison iliotibial-band transfer for a torn anterior cruciate ligament of the knee. Long-term follow-up. J Bone Joint Surg Am 1992;74:1392-402.

57. Rockborn P, Gillquist J. Outcome of arthroscopic meniscectomy. a 13-year physical and radiographic follow-up of 43 patients under 23 years of age. Acta Orthop Scand 1995;66:113-7.

58. Rockborn P, Gillquist J. Results of open meniscus repair. Long-term follow-up study with a matched uninjured Control group. J Bone Joint Surg Br 2000;82:494-8.

59. Roos EM, Bremander $A B$, Englund $M$, et al. Change in self-reported outcomes and objective physical function over 7 years in middle-aged subjects with or at high risk of knee osteoarthritis. Ann Rheum Dis 2008;67:505-10.

60. Sanders TL, Pareek A, Kremers HM, et al. Long-term follow-up of isolated ACL tears treated without ligament reconstruction. Knee Surg Sports Traumatol ArthrosC 2017:25:493-500.

61. Scheller G, Sobau C, Bülow JU. Arthroscopic partial lateral meniscectomy in an otherwise normal knee: clinical, functional, and radiographic results of a long-term follow-up study. Arthroscopy 2001;17:946-52.

62. Segawa H, Omori G, Koga Y. Long-term results of non-operative treatment of anterior cruciate ligament injury. The Knee 2001;8:5-11.

63. Sommerlath K, Lysholm J, Gillquist J. The long-term course after treatment of acute anterior cruciate ligament ruptures. Am J Sports Med 1991;19:156-62.

64. Sonne-Holm S, Fledelius I, Ahn NCN. Results after meniscectomy in 147 athletes. Acta Orthopaedica Scandinavica 1980;51:303-9.

65. Stein T, Mehling AP, Welsch F, et al. Long-term outcome after arthroscopic meniscal repair versus arthroscopic partial meniscectomy for traumatic meniscal tears. Am J Sports Med 2010;38:1542-8.

66. Strand T, Mølster A, Hordvik M, et al. Long-term follow-up after primary repair of the anterior cruciate ligament: clinical and radiological evaluation 15-23 years postoperatively. Arch Orthop Trauma Surg 2005;125:217-21.

67. Sun K, Tian S-qi, Zhang J-hua, et al. Anterior cruciate ligament reconstruction with bone-patellar tendon-bone autograft versus allograft. Arthroscopy 2009;25:750-9.

68. Sun K, Zhang J, Wang Y, et al. A prospective randomized comparison of irradiated and non-irradiated hamstring tendon allograft for $\mathrm{ACL}$ reconstruction. Knee Surg Sports Traumatol Arthrosc 2012;20:187-94.

69. Sutherland AG, Cooper K, Alexander LA, et al. The long-term functional and radiological outcome after open reconstruction of the anterior cruciate ligament. J Bone Joint Surg Br 2010;92-B:1096-9.

70. van der Hart CP, van den Bekerom MPJ, Patt TW. The occurrence of osteoarthritis at a minimum of ten years after reconstruction of the anterior cruciate ligament. J Orthop Surg Res 2008;3.

71. Yamaguchi S, Sasho T, Tsuchiya A, et al. Long term results of anterior cruciate ligament reconstruction with iliotibial tract: 6-, 13-, and 24-year longitudinal follow-up. Knee Surg Sports Traumatol Arthrosc 2006;14:1094-100. 